\title{
Magnetic Field Induced Quantum Spin Liquid in the Two Coupled Trillium Lattices of $\mathrm{K}_{2} \mathrm{Ni}_{2}\left(\mathrm{SO}_{4}\right)_{3}$
}

\author{
Ivica Živković $\odot,{ }^{1, *}$ Virgile Favre $\odot,{ }^{1}$ Catalina Salazar Mejia $\odot,{ }^{2}$ Harald O. Jeschke $\odot,{ }^{3}$ Arnaud Magrez, ${ }^{4}$ \\ Bhupen Dabholkar@, ${ }^{5}$ Vincent Noculak, ${ }^{6,7}$ Rafael S. Freitas $\odot,{ }^{8}$ Minki Jeong $\odot,{ }^{9}$ Nagabhushan G. Hegde, ${ }^{1}$ Luc Testa๑, ${ }^{1}$ \\ Peter Babkevich, ${ }_{1}^{1}$ Yixi Su $\odot,{ }^{10}$ Pascal Manuel, ${ }^{11}$ Hubertus Luetkens, ${ }^{12}$ Christopher Baines, ${ }^{12}$ Peter J. Baker $\odot,{ }^{11}$ \\ Jochen Wosnitza, ${ }^{2,13}$ Oksana Zaharko, ${ }^{14}$ Yasir Iqbal $\odot,{ }^{5}$ Johannes Reuther, ${ }^{6,7}$ and Henrik M. Rønnow ${ }^{1}$ \\ ${ }^{1}$ Laboratory for Quantum Magnetism, Institute of Physics, École Polytechnique Fédérale de Lausanne, \\ CH-1015 Lausanne, Switzerland \\ ${ }^{2}$ Hochfeld-Magnetlabor Dresden (HLD-EMFL) and Würzburg-Dresden Cluster of Excellence ct.qmat, \\ Helmholtz-Zentrum Dresden-Rossendorf, 01328 Dresden, Germany \\ ${ }^{3}$ Research Institute for Interdisciplinary Science, Okayama University, Okayama 700-8530, Japan \\ ${ }^{4}$ Crystal Growth Facility, École Polytechnique Fédérale de Lausanne, CH-1015 Lausanne, Switzerland \\ ${ }^{5}$ Department of Physics and Quantum Centers in Diamond and Emerging Materials (QuCenDiEM) Group, \\ Indian Institute of Technology Madras, Chennai 600036, India \\ ${ }^{6}$ Dahlem Center for Complex Quantum Systems and Fachbereich Physik, Freie Universität Berlin, 14195 Berlin, Germany \\ ${ }^{7}$ Helmholtz-Zentrum für Materialien und Energie, Hahn-Meitner-Platz 1, 14109 Berlin, Germany \\ ${ }^{8} 7$ Instituto de Física, Universidade de São Paulo, 05508-090 São Paulo, Brazil \\ ${ }^{9}$ School of Physics and Astronomy, University of Birmingham, Edgbaston, Birmingham B15 2TT, United Kingdom \\ ${ }^{10}$ Jülich Centre for Neutron Science (JCNS) at Heinz Maier-Leibnitz. Zentrum (MLZ), \\ Forschungszentrum Jülich, Lichtenbergstrasse 1, D-85747 Garching, Germany \\ ${ }^{11}$ ISIS Pulsed Neutron and Muon Source, STFC Rutherford Appleton Laboratory, \\ Harwell Science and Innovation Campus, Didcot, Oxfordshire OX11 OQX, United Kingdom \\ ${ }^{12}$ Laboratory for Muon Spin Spectroscopy, Paul Scherrer Institute, CH-5232 Villigen, Switzerland \\ ${ }^{13}$ Institut für Festkörper- und Materialphysik, TU Dresden, 01062 Dresden, Germany \\ ${ }^{14}$ Laboratory for Neutron Scattering and Imaging, Paul Scherrer Institut, CH-5253 Villigen, Switzerland
}

(Received 3 May 2021; revised 4 August 2021; accepted 8 September 2021; published 6 October 2021)

\begin{abstract}
Quantum spin liquids are exotic states of matter that form when strongly frustrated magnetic interactions induce a highly entangled quantum paramagnet far below the energy scale of the magnetic interactions. Three-dimensional cases are especially challenging due to the significant reduction of the influence of quantum fluctuations. Here, we report the magnetic characterization of $\mathrm{K}_{2} \mathrm{Ni}_{2}\left(\mathrm{SO}_{4}\right)_{3}$ forming a threedimensional network of $\mathrm{Ni}^{2+}$ spins. Using density functional theory calculations, we show that this network consists of two interconnected spin-1 trillium lattices. In the absence of a magnetic field, magnetization, specific heat, neutron scattering, and muon spin relaxation experiments demonstrate a highly correlated and dynamic state, coexisting with a peculiar, very small static component exhibiting a strongly renormalized moment. A magnetic field $B \gtrsim 4 \mathrm{~T}$ diminishes the ordered component and drives the system into a pure quantum spin liquid state. This shows that a system of interconnected $S=1$ trillium lattices exhibits a significantly elevated level of geometrical frustration.
\end{abstract}

DOI: 10.1103/PhysRevLett.127.157204

Strongly correlated systems are at the forefront of condensed matter research, exhibiting exotic phases and nourishing novel theoretical concepts. In magnetism, one of the most sought-after strongly correlated phases is a quantum spin liquid (QSL), a state in which spins avoid long-range order (LRO) and are considered entangled on all spatial scales [1-3]. To realize a QSL, the geometrical frustration and reduced dimensionality of the magnetic subsystem have been considered vital. One-dimensional Heisenberg chains exhibit QSL behavior even without frustration [4,5], while three-dimensional (3D) cases are rare due to the significant reduction of quantum fluctuations. Nevertheless, it has been found that 3D lattices like pyrochlore [6-8] and hyperhyperkagome $[9,10]$ support QSL behavior.

In this Letter, we provide extensive experimental and computational evidence that $\mathrm{K}_{2} \mathrm{Ni}_{2}\left(\mathrm{SO}_{4}\right)_{3}$ exhibits QSL behavior, based on a novel arrangement of spins forming two interconnected trillium lattices. Previous work on compounds featuring a single trillium lattice was mainly driven by a pressure-induced quantum phase transition discovered in the itinerant helimagnet $\mathrm{MnSi}$ [11] and evidence of non-Fermi liquid behavior above a critical pressure [12]. Later theoretical works [13,14] showed some degree of geometrical frustration in the trillium lattice that 
(a)

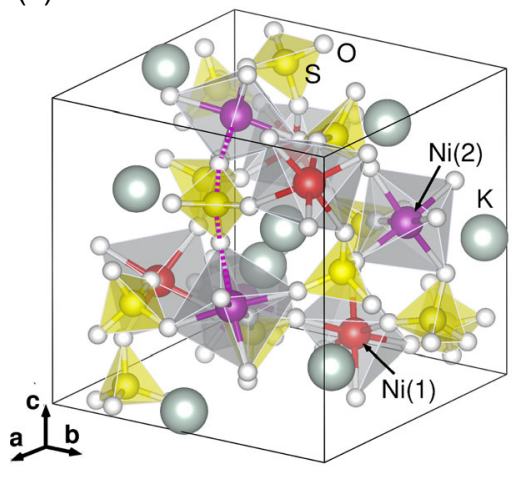

(b)

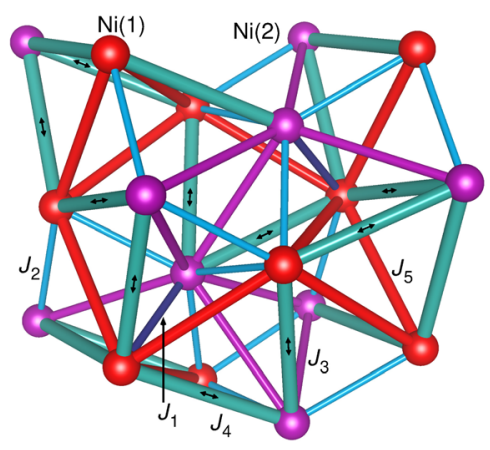

(c)

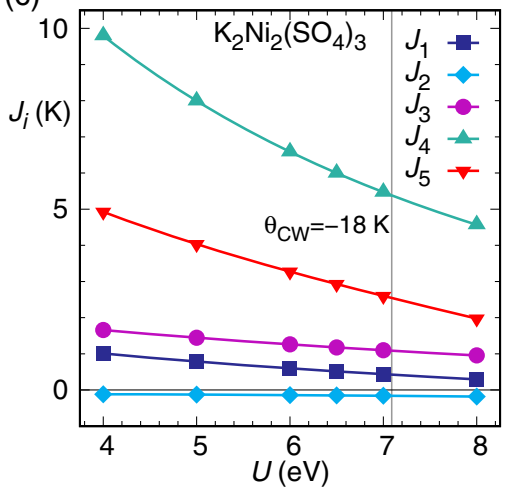

FIG. 1. (a) Unit cell of $\mathrm{K}_{2} \mathrm{Ni}_{2}\left(\mathrm{SO}_{4}\right)_{3}$. A Ni-O-S-O-Ni super-superexchange path contributing to a trillium coupling is marked by dashed lines. (b) Exchange network between nickel sites. A ten-site loop formed by the strongest exchange $J_{4}$ is marked by arrows. (c) Exchange couplings determined by DFT energy mapping. The vertical line indicates the $U$ value where the calculated Curie-Weiss temperature matches the experimental value.

was however insufficient to prevent the onset of LRO. From that perspective, $\mathrm{K}_{2} \mathrm{Ni}_{2}\left(\mathrm{SO}_{4}\right)_{3}$ and other members of the langbeinite family $\mathrm{K}_{2} \mathrm{M}_{2}\left(\mathrm{SO}_{4}\right)_{3}(M=\mathrm{Fe}$, Co, $\mathrm{Mn}, \mathrm{Cr}$ ) offer an arena for testing future theoretical developments on interconnected trillium lattices. Previous investigations of those compounds displayed ferroelectricity and structural transitions but their magnetic properties remain terra incognita.

$\mathrm{K}_{2} \mathrm{Ni}_{2}\left(\mathrm{SO}_{4}\right)_{3}$ crystallizes in a cubic unit cell $\left(P 2_{1} 3\right)$ with $a=9.81866(12) \AA$ determined from single-crystal diffraction at $100 \mathrm{~K}$ [15]. It consists of a network of trigonally distorted $\mathrm{NiO}_{6}$ octahedra, coupled through $\mathrm{SO}_{4}$ groups [Fig. 1(a)], with a Ni-O-S-O-Ni super-superexchange mechanism mediating magnetic interactions between $S=1$ spins. There are two crystallographic Ni sites, distinguished by their $\mathrm{Ni}-\mathrm{O}$ distances [15], each site forming a single trillium lattice.

Mapping the generalized gradient approximation with Hubbard $U$ parameter total energies [15] onto a Heisenberg Hamiltonian for $\mathrm{K}_{2} \mathrm{Ni}_{2}\left(\mathrm{SO}_{4}\right)_{3} \hat{\mathcal{H}}=\sum_{i<j} J_{i j} \hat{\mathbf{S}}_{i} \cdot \hat{\mathbf{S}}_{j}$ as shown in Fig. 1(c) yields the five nonzero exchange couplings that are listed in Table I and shown in Fig. 1(b), visualizing the exchange network. The couplings within each trillium lattice are given by antiferromagnetic (AFM) $J_{3}$ and $J_{5}$, respectively. On the other hand, the strongest coupling is found to be AFM $J_{4}$ that interconnects the two lattices.

TABLE I. $\mathrm{K}_{2} \mathrm{Ni}_{2}\left(\mathrm{SO}_{4}\right)_{3}$ exchange energies obtained by DFT energy mapping, with paths identified by $\mathrm{Ni}-\mathrm{Ni}$ distance.

\begin{tabular}{lccc}
\hline \hline Label & Type & Distance $(\AA)$ & Exchange $(\mathrm{K})$ \\
\hline$J_{1}$ & $\mathrm{Ni}(1)-\mathrm{Ni}(2)$ & 4.42877 & $0.42(1)$ \\
$J_{2}$ & $\mathrm{Ni}(1)-\mathrm{Ni}(2)$ & 4.90057 & $-0.16(1)$ \\
$J_{3}$ & $\mathrm{Ni}(2)-\mathrm{Ni}(2)$ & 6.08379 & $1.09(1)$ \\
$J_{4}$ & $\mathrm{Ni}(1)-\mathrm{Ni}(2)$ & 6.12050 & $5.38(1)$ \\
$J_{5}$ & $\mathrm{Ni}(1)-\mathrm{Ni}(1)$ & 6.12695 & $2.54(1)$ \\
\hline \hline
\end{tabular}

Interestingly, if $J_{4}$ were the only coupling in the system, it would support a Néel-type LRO. Thus, our calculation shows that the physics of $\mathrm{K}_{2} \mathrm{Ni}_{2}\left(\mathrm{SO}_{4}\right)_{3}$ is determined by an interplay between $J_{4}$ induced ordering tendencies and $J_{3}$ and $J_{5}$ driven frustration.

Figure 2(a) displays the temperature dependence of dc magnetic susceptibility $\chi_{\mathrm{dc}}(T)$ and its inverse in a wide temperature range. The monotonic increase of $\chi_{\mathrm{dc}}(T)$ with decreasing $T$, without any noticeable features, suggests the absence of LRO down to $2 \mathrm{~K}$. The linear behavior of $1 / \chi_{\mathrm{dc}}(T)$ above $50 \mathrm{~K}$ allows us to use the Curie-Weiss law $\chi(T)=C /\left(T-\Theta_{\mathrm{CW}}\right)$, which gives $C=1.37(2) \mathrm{emu} \mathrm{K} / \mathrm{mol}$ and $\Theta_{\mathrm{CW}}=-18(1) \mathrm{K}$. The value of $C$ corresponds to $S=1$ with a slightly enhanced $g$ factor of $g=2.34$. $\Theta_{\mathrm{CW}}<0$ indicates predominant AFM interactions in accordance with density functional theory (DFT) calculations. Additionally, measurements along three orthogonal directions practically overlap, indicating no significant anisotropy.

Below $50 \mathrm{~K}, 1 / \chi_{\mathrm{dc}}(T)$ starts to deviate from the CurieWeiss law, following the buildup of correlations between magnetic moments. To emphasize this behavior, magnetization curves obtained at several temperatures are plotted in Fig. 2(b), together with the curves of the Brillouin function, which describe an assembly of noninteracting $S=1$ spins, at corresponding temperatures (dashed lines). To approximate the magnetization behavior, classical Monte Carlo calculations employing the DFT Hamiltonian have been performed. The resulting curves (full lines) are closer to the experimental ones, but it is apparent that with decreasing $T$ the deviation from the classical prediction becomes more pronounced, suggesting a sizeable influence of quantum fluctuations on this 3D lattice.

Further evidence of strongly correlated spins can be obtained from specific heat measurements. Figure 2(c) shows the $T$ dependence of the total specific heat of a single crystal of $\mathrm{K}_{2} \mathrm{Ni}_{2}\left(\mathrm{SO}_{4}\right)_{3}$, together with a nonmagnetic 
(a)

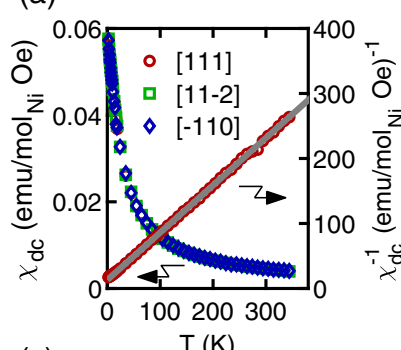

(c)

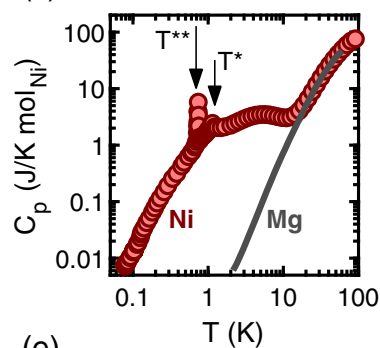

(e)

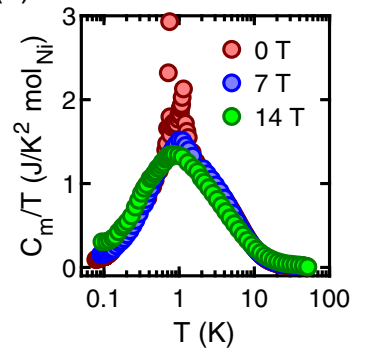

(b)

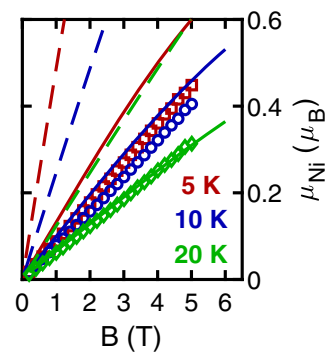

(d)

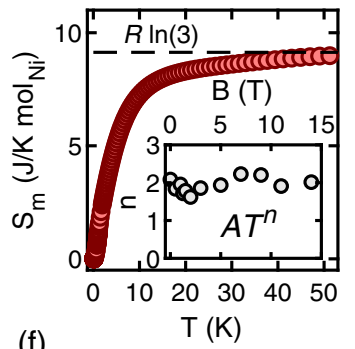

(f)

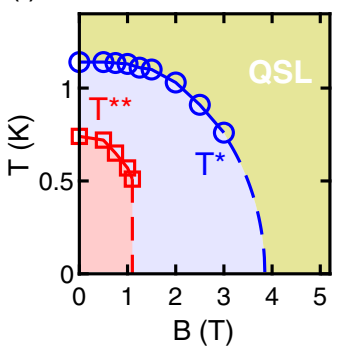

FIG. 2. (a) $T$ dependence of $\chi_{\mathrm{dc}}$ along the three orthogonal directions (left axis) for $B=0.1 \mathrm{~T}$, and the inverse susceptibility $1 / \chi_{\text {dc }}$ (right axis) with $B \|[111]$. The solid gray line represents the Curie-Weiss law. (b) Magnetic field dependence of magnetization, together with the Brillouin function for $S=1$ and $g=2.34$ (dashed lines) and Monte Carlo simulations (solid lines). (c) Zero field temperature dependence of the total specific heat of $\mathrm{K}_{2} \mathrm{Ni}_{2}\left(\mathrm{SO}_{4}\right)_{3}$, together with the total specific heat of $\mathrm{K}_{2} \mathrm{Mg}_{2}\left(\mathrm{SO}_{4}\right)_{3}$ representing the phonon contribution. (d) $T$ dependence of magnetic entropy. An inset shows the magnetic field dependence of the exponent $n$ in the power law $C_{p}=A T^{n}$. (e) $T$ dependence of the magnetic specific heat for several magnetic field values. (f) $T-B$ phase diagram of $\mathrm{K}_{2} \mathrm{Ni}_{2}\left(\mathrm{SO}_{4}\right)_{3}$.

analog $\mathrm{K}_{2} \mathrm{Mg}_{2}\left(\mathrm{SO}_{4}\right)_{3}$. At temperatures above $20 \mathrm{~K}$, the two compounds show a very similar behavior, indicating a dominant phonon contribution. Below $20 \mathrm{~K}, \mathrm{~K}_{2} \mathrm{Ni}_{2}\left(\mathrm{SO}_{4}\right)_{3}$ exhibits a significant deviation, with a broad maximum around $5 \mathrm{~K}$ and two features occurring at $T^{*}=1.14 \mathrm{~K}$ and $T^{* *}=0.74 \mathrm{~K}$. Below $T^{* *}$, the heat capacity behaves according to a power law $C_{p} \sim T^{n}$, with $n \approx 2$. This value of the exponent differs appreciably from $n=3$ for classical AFM and has been observed in several frustrated magnetic systems [8,26-28].

To extract the magnetic specific heat $C_{m}$, the phonon contribution using the data obtained on $\mathrm{K}_{2} \mathrm{Mg}_{2}\left(\mathrm{SO}_{4}\right)_{3}$ has been subtracted. On the high $T$ side the subtraction works up to $50 \mathrm{~K}$, where $\mathrm{K}_{2} \mathrm{Mg}_{2}\left(\mathrm{SO}_{4}\right)_{3}$ shows a kink [15] associated with previously observed lattice related features

in the heat capacity [29]. On the low $T$ side, a polynomial $B T^{3}+C T^{5}$ has been used [15]. The error of the total entropy $S=\int\left(C_{m} / T\right) d T$ due to background subtraction is estimated to be a few percent. As can be seen in Fig. 2(d), at $50 \mathrm{~K}$ more than $98 \%$ of the expected entropy for $S=1$ system is recovered, with more than $90 \%$ being released up to $20 \mathrm{~K}$. The saturation toward the $R \ln (2 S+1)$ value for $S=1$ indicates that no residual entropy is present at $T=0$ and that $\mathrm{K}_{2} \mathrm{Ni}_{2}\left(\mathrm{SO}_{4}\right)_{3}$ exhibits a nondegenerate ground state.

Application of a magnetic field along the [111] direction induces little change in the overall behavior of the heat capacity of $\mathrm{K}_{2} \mathrm{Ni}_{2}\left(\mathrm{SO}_{4}\right)_{3}$. A small but noticeable redistribution occurs for fields above $B=7 \mathrm{~T}$ [Fig. 2(e)], but even with fields up to $14 \mathrm{~T}$ the overall shape of the curve remains unchanged. The power law $C_{p} \sim T^{n}$ observed at low $T$ for $B=0$ is maintained for $B>0$ without a visible crossover toward the gapped polarized state, as seen, for example, in $\mathrm{YbMgGaO}_{4}$ [30]. The value of the extracted exponent remains field independent up to $14 \mathrm{~T}$ [inset of Fig. 2(d)].

The order of transitions at $T^{*}$ and $T^{* *}$ is revealed through their overall shape. The feature at $T^{*}$ resembles a typical, asymmetric $\lambda$ shape, characteristic of second-order phase transitions. On the other hand, at $T^{* *}$ a narrow, symmetrical peak is found, often seen in first-order phase transitions. Although the entropy released at $T^{*}$ amounts to only $1 \%$ of the total $R \ln 3$ [15], the sample purity determined by single-crystal $x$-ray diffraction [15] rules out any impurityrelated scenario. Additionally, a comparison with specificheat measurements on a powder sample reveals that $T^{*}$ is significantly diminished while $T^{* *}$ is completely absent [15]. With a tentative assignment of $T^{* *}$ as a first-order phase transition, its presence in a single-crystal experiment suggests that it is intrinsically related to the lowtemperature magnetic phase of $\mathrm{K}_{2} \mathrm{Ni}_{2}\left(\mathrm{SO}_{4}\right)_{3}$.

The magnetic field dependence of $T^{*}$ and $T^{* *}$ is presented in Fig. 2(f). $T^{* *}$ is quickly diminished in amplitude and for $B>1 \mathrm{~T}$ it disappears completely. $T^{*}$ is practically unchanged up to $B=1 \mathrm{~T}$, with a subsequent decrease and a reduction of the size of the anomaly [15]. By assuming a quadratic $B$ dependence of the second-order phase transition, the value of the critical magnetic field $B_{c} \lesssim 4 \mathrm{~T}$ can been estimated, above which a completely dynamic and fluctuating state exists down to the lowest $T$.

To shed more light on the peculiar magnetic properties of $\mathrm{K}_{2} \mathrm{Ni}_{2}\left(\mathrm{SO}_{4}\right)_{3}$, a series of neutron scattering experiments have been performed. Figure 3(a) shows the results of polarized neutron scattering in which a $Q$ dependence of the scattering intensity at $0.5 \mathrm{~K}$ is presented. It exhibits a broad maximum centered at $Q_{\max } \approx 0.75 \AA^{-1}$, followed by an attenuating oscillatory dependence. Such a broad, liquidlike structure factor is typical for systems with strong quantum fluctuations. This conclusion is further supported by the fact that the diffuse scattering pattern in Fig. 3(a) is 

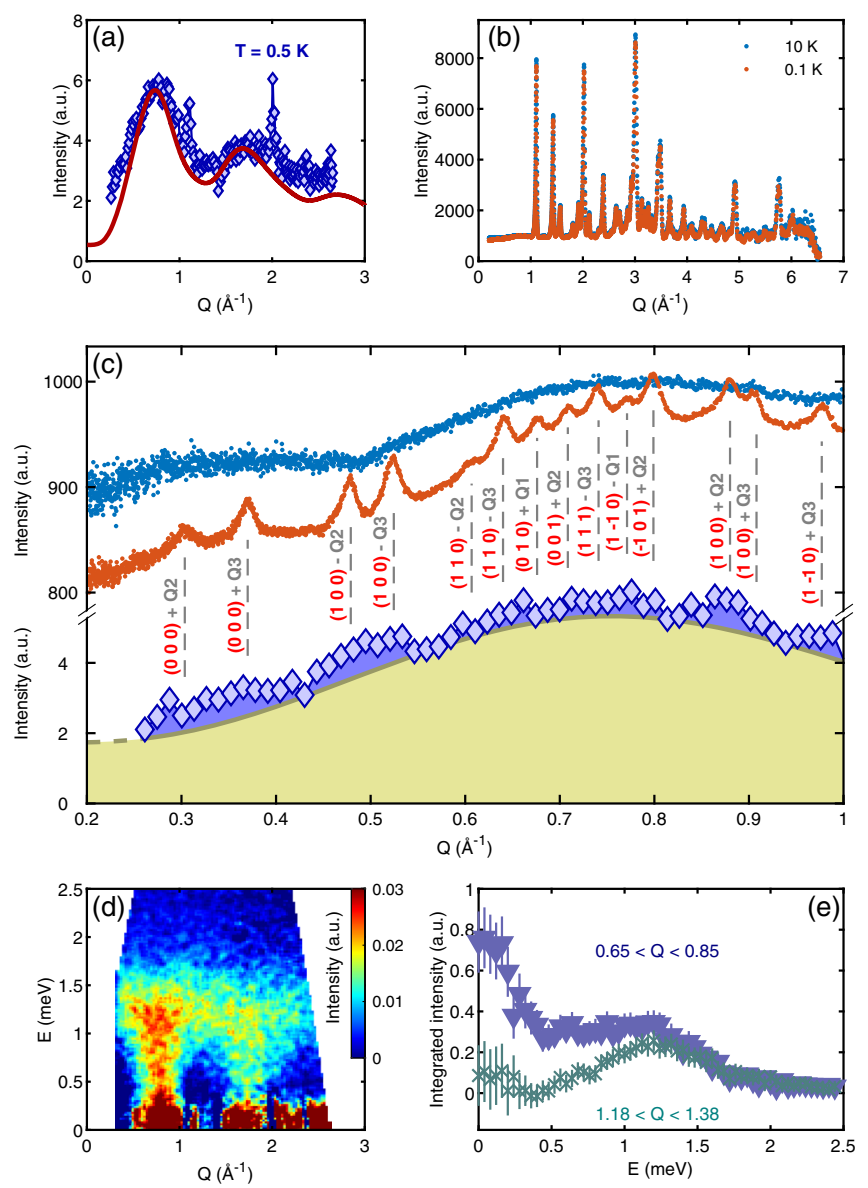

FIG. 3. (a) Pure magnetic scattering pattern derived from the spin-polarized neutron diffraction data at $0.5 \mathrm{~K}$ (blue diamonds) and from pseudofermion functional renormalization group simulation (solid line). (b) Neutron powder diffraction at $0.1 \mathrm{~K}$ and $10 \mathrm{~K}$. (c) Combined data from polarized neutrons [blue diamonds from panel (a)] and powder diffraction [blue $(10 \mathrm{~K})$ and red points $(0.1 \mathrm{~K})$ from panel (b)]. Blue shading indicates an upper limit of the contribution from the ordered component, yellow shading represents the contribution from the fluctuating component, (d) Time-of-flight data at $0.5 \mathrm{~K}$. (e) $E$ dependence of the integrated intensity for two $Q$ ranges.

well reproduced by pseudofermion functional renormalization group simulations of the DFT Hamiltonian. Remarkably, despite the general difficulties in simulating a strongly fluctuating 3D spin system with complex frustrated interactions as realized in $\mathrm{K}_{2} \mathrm{Ni}_{2}\left(\mathrm{SO}_{4}\right)_{3}$, not only the positions of the extrema are well reproduced but also the global amplitude variations. Additionally, the oscillatory behavior is seen to persist at least up to $17 \mathrm{~K}$ [15], clearly indicating its connection to strong correlations developing below $20 \mathrm{~K}$.

To investigate the system's static component, a neutron powder diffraction experiment has been performed well above and well below $T^{*}$. From a wide $Q$ diffraction pattern shown in Fig. 3(b), it is found that for $Q>1 \AA^{-1}$ all peaks are present at both temperatures, indicating their lattice origin. On the other hand, a series of very weak magnetic peaks can be found at $T=0.1 \mathrm{~K}$ for $Q<1 \AA^{-1}$, as seen in the upper half of Fig. 3(c). They can all be assigned to satellites of the main nuclear Bragg peaks $(h, k, l)$ in the form $q_{\text {magnetic }}=(h, k, l) \pm Q_{i}$, where $Q_{1}=\left(\frac{1}{3}, 0,0\right)$, $Q_{2}=\left(\frac{1}{3}, \frac{1}{3}, 0\right)$, and $Q_{3}=\left(\frac{1}{3}, \frac{1}{3}, \frac{1}{3}\right)$. The existence of three propagation vectors indicates that even LRO is heavily influenced by frustration, leaving several possible structures with similar ground state energies.

Because of the complexity of the scattering pattern, including several propagation vectors, the tripling of the magnetic unit cell, and very weak amplitudes, it is not possible to completely determine the magnetic structure or to extract the value of the ordered moment. Nevertheless, using a purely magnetic scattering pattern from polarized neutrons [Fig. 3(a)], one can estimate an upper limit for the static component. To this end, we envisage that the total intensity $S(Q)$ is composed of two contributions $S(Q)=$ $S_{\text {static }}(Q)+S_{\text {dynamic }}(Q)$, with the jagged $S_{\text {static }}(Q)$ roughly following the powder diffraction profile and sitting on top of the smooth $S_{\text {dynamic }}(Q)$. Although the resultant ratio $S_{\text {static }}(Q) / S(Q) \approx 11 \%$ cannot be directly related to the value of the ordered moment, it serves as supporting evidence that the ground state in $\mathrm{K}_{2} \mathrm{Ni}_{2}\left(\mathrm{SO}_{4}\right)_{3}$ is dominated by spin fluctuations.

In Fig. 3(d), we show time-of-flight data obtained as a direct subtraction of the background intensity obtained at $80 \mathrm{~K}$ from a measured intensity at $0.5 \mathrm{~K}$. Streaks of intensity can be observed at the same positions as maxima in $S(Q)$ found with polarized neutrons. The upper limit of spin excitations is found to be around $1.8 \mathrm{meV}$, which agrees well with the temperature at which specific heat starts to significantly deviate from a purely phononic behavior. In Fig. 3(e), a narrow Q-integrated energy dependence of intensity is shown, indicating a continuum of excitations down to the elastic line. Because of the existence of the ordered component, it is not straightforward to assign this continuum to the QSL state. On the other hand, the dominance of the dynamic component, revealed by specific heat data and polarized neutron scattering, renders this conclusion very plausible, which would then support the hypothesis of a gapless nature for the QSL.

To probe further the peculiar coexistence of static and dynamic properties revealed in $\mathrm{K}_{2} \mathrm{Ni}_{2}\left(\mathrm{SO}_{4}\right)_{3}$, muon spin relaxation $(\mu \mathrm{SR})$ experiments have been performed. As shown in Fig. 4(a), no obvious wiggles are observed down to lowest $T$. On a phenomenological level, the relaxation is often described by a stretched-exponential function

$$
A(t)=A_{0} e^{-(\lambda t)^{\beta}}+A_{\mathrm{BG}},
$$

where $A_{0}$ is the initial asymmetry, $A_{\mathrm{BG}}$ a constant background, $\lambda$ is the relaxation rate, and $\beta$ is the stretching exponent that in an ideal case of $\beta=1$ leads to a simple 

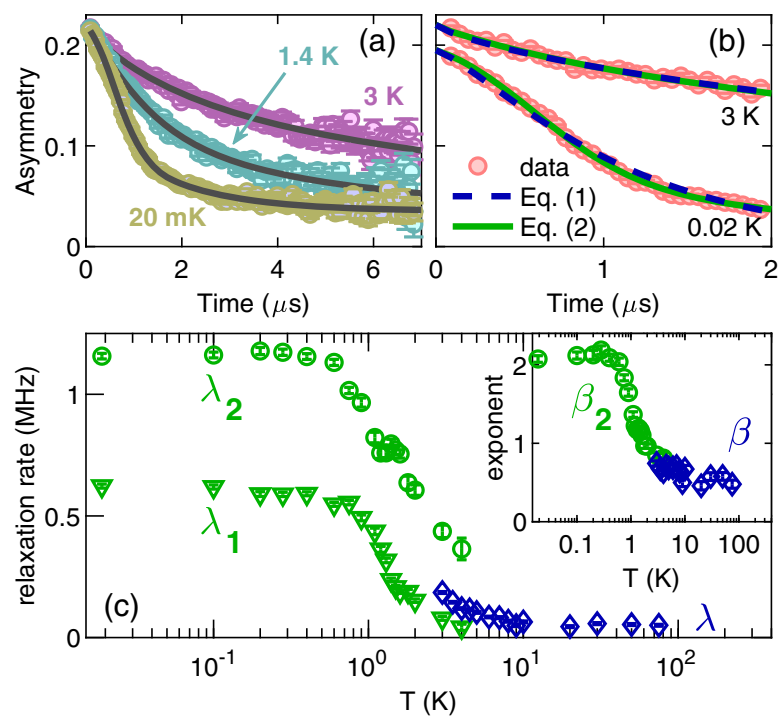

FIG. 4. (a) ZF- $\mu$ SR spectra at selected temperatures. The solid lines represent fitted curves following the model in Eq. (2). (b) A comparison of the applicability of two models at $0.02 \mathrm{~K}$ (shifted down for clarity) and $3 \mathrm{~K}$. (c) Temperature dependence of the $\mu \mathrm{SR}$ rates extracted from two models. Circles and triangles represent relaxation rates from the model in Eq. (2), diamonds from the model in Eq. (1). The inset displays the temperature dependence of the exponent $\beta_{2}$ from Eq. (2) and $\beta$ from Eq. (1).

exponential relaxation. $\beta<1$ has usually been associated with either a distribution of relaxation times, multiple muon stopping sites, or with intrinsic disorder in the magnetic system. As is evident from Fig. 4(b), at low $T$ the observed time dependence of the asymmetry cannot be satisfactorily described by a single contribution. Thus, we have extended Eq. (1) with an additional term,

$$
A(t)=A_{0}\left(f e^{-\left(\lambda_{1} t\right)^{\beta_{1}}}+(1-f) e^{-\left(\lambda_{2} t\right)^{\beta_{2}}}\right)+A_{\mathrm{BG}},
$$

and fixed $f=0.5$ and $\beta_{1}=1$ to avoid overparameterization. We find that it is necessary to use Eq. (2) up to $3 \mathrm{~K}$, while for $T>3 \mathrm{~K}$, Eq. (1) is sufficient (for the discussion of the overlapping region, see [15]). In Fig. 4(c), we present the temperature evolution of relaxation rates and exponents (see inset) extracted using Eq. (2) (green symbols) and Eq. (1) (blue symbols).

Below $T \sim 1 \mathrm{~K}$, the extracted parameters attain a constant value, a feature often associated with a highly dynamic nature of QSLs [31-33]. We point out that the value of the exponent $\beta \simeq 2$ is indicative of a specific type of a correlated spin system based on spin singlets [34]. Within this scenario, the Gaussian shape of the relaxation profile develops from a sporadic appearance of unpaired spins. The time interval of their existence is much shorter than a lifetime of a muon, so for the majority of time muons experience very small fields related to the short-lived but very distant unpaired spins. Such a scenario is in accordance with a practically field-independent magnetic specific heat seen in Fig. 2(e) [26]. Within this framework, the strong relaxation at low temperatures described by $\lambda_{1}$ can be associated with a partial but homogeneous order, while the remaining dynamics is due to the sporadic unpaired-spin appearances. The absence of oscillations can then be associated with a spread of local fields originating from complex magnetic structures given by propagation vectors $Q_{1}, Q_{2}$, and $Q_{3}$. Additionally, the coherent regions giving rise to magnetic peaks in neutron diffraction are probed on much shorter timescales $\left(\sim 10^{-14} \mathrm{~s}\right)$, allowing for local fluctuations between different magnetic structures on the timescale of muons.

We find two possible scenarios that could encompass a small value of the static component existing alongside the dominant, fluctuating component. The first scenario assumes the existence of a quantum critical point (QCP) between an ordered phase and a quantum fluctuationdominant phase, with $\mathrm{K}_{2} \mathrm{Ni}_{2}\left(\mathrm{SO}_{4}\right)_{3}$ being on the ordered side of QCP but "accidentally" close to it. In this case, the ordered moment $m_{s}$ is strongly renormalized due to the prevalence of quantum fluctuations close to a QCP, as has been demonstrated in $\mathrm{TlCuCl}_{3}$ where a pressure-controlled quantum phase transition between a LRO AFM state and a nonmagnetic dimer phase is arbitrarily decreased $\left(m_{s} \sim \sqrt{p-p_{c}}\right)$ close to a QCP [35]. In this context, a possible control parameter could be the ratio of intratrillium $\left(J_{3}, J_{5}\right)$ and intertrillium $\left(J_{1}, J_{2}, J_{4}\right)$ lattice couplings. Given that $\mathrm{Ni}(1)$ and $\mathrm{Ni}(2)$ sites form a bipartite lattice, the limit of dominant $J_{1}, J_{2}, J_{4}$ results in a semiclassical AFM phase. With $J_{3}, J_{5}$ dominant, the system is in the limit of two weakly coupled trillium lattices. As demonstrated theoretically for a single trillium lattice, such a system is expected to form a variant of the $120^{\circ}$ order $[13,14]$. The case of two interconnected trillium lattices represents a novel research direction with many members of the langbeinite family providing ample opportunity for comparison with theory.

The second scenario dismisses the "fortuitous" constellation of parameters describing $\mathrm{K}_{2} \mathrm{Ni}_{2}\left(\mathrm{SO}_{4}\right)_{3}$ and considers it positioned well within the QSL phase. Because of the presence of the antisymmetric exchange coupling (the Dzyaloshinskii-Moriya interaction) allowed by the noncentrosymmetric space group, the ground state gets "dressed" with a small ordered component due to the admixing of higher lying states, similar to the admixture of triplet wave functions into the ground state singlet of an AFM dimer. An exciting consequence of this scenario arises from topological aspects imposed on the QSL state. Magnetic structures forming in noncentrosymmetric space groups are shown to support skyrmions, which are topologically protected spin textures [36,37]. The fractional wave numbers $Q_{1}, Q_{2}$, and $Q_{3}$ revealed in the diffraction experiment do indicate a potential role of the Dzyaloshinskii-Moriya interaction in the formation of LRO. 
In either case, the observed coexistence between fluctuating spins and a small static component that vanishes in a magnetic field could be linked to already developed concepts like field-induced spin liquids in Kitaev-type honeycomb models featuring non-Abelian fractional quasiparticles [38]. The ability to tune its behavior across a QCP with a magnetic field into a pristine QSL state is an exciting opportunity that should stimulate further experimental and theoretical studies.

All the data files needed to plot figures presented in the paper can be found in [39].

We thank Bi Wen Hua for his help with x-ray diffraction experiments. I. $\check{Z}$. acknowledges financial support by the Swiss National Science Foundation (SNSF) Projects No. 200021-169699 and 206021-189644. H. M. R. acknowledges financial support by SNFS Projects No. 200020-188648 and 206021-189644. Part of this work was supported by the Deutsche Forschungsgemeinschaft (DFG) through the SFB 1143 and the Würzburg-Dresden Cluster of Excellence on Complexity and Topology in Quantum Matter-ct: qmat (EXC 2147, Project No. 390858490), the Innovation Pool project MaDQuanT, as well as by the HLD at HZDR, a member of the European Magnetic Field Laboratory (EMFL). R.S.F. acknowledges financial support by FAPESP (Grant No. 2015/16191-5) and CNPq (Grant No. 429511/2018-3). J. R. acknowledges financial support by the German Research Foundation within the CRC183 (project A04). Y. I. acknowledges financial support by SERB, Department of Science and Technology (DST), India through grants SRG (No. SRG/2019/000056), MATRICS (No. MTR/2019/001042), and Indo-French Center for the Promotion of Advanced Research CEFIPRA (No. 64T3-1). This research was supported in part by the National Science Foundation under Grant No. NSF PHY-1748958, the ICTP through the Simons Associateship scheme, IIT Madras through the IoE program for establishing the QuCenDiEM group (Project No. SB20210813PHMHRD002720), and ICTS, Bengaluru, India during a visit for participating in the program "Novel phases of quantum matter" (Code: ICTS/ topmatter2019/12). Y. I. acknowledges the use of the computing resources at HPCE, IIT Madras. The $\mu \mathrm{SR}$ experiments were performed at the MUSR beamline [ISIS (1710223)] and the LTF and GPS beamlines [PSI (2017119 and 2017119)]. The neutron diffraction experiment was performed at WISH beamline [ISIS (2010010)]. The spin-polarized neutron diffraction and time-of-flight experiments were performed at DNS beamline [MLZ (13656)].

*Corresponding author. ivica.zivkovic@epfl.ch
[1] L. Savary and L. Balents, Quantum spin liquids: A review, Rep. Prog. Phys. 80, 016502 (2017).

[2] Y. Zhou, K. Kanoda, and T.-K. Ng, Quantum spin liquid states, Rev. Mod. Phys. 89, 025003 (2017).

[3] C. Broholm, R. J. Cava, S. A. Kivelson, D. G. Nocera, M. R. Norman, and T. Senthil, Quantum spin liquids, Science 367, eaay0668 (2020).

[4] B. Lake, D. A. Tennant, J. S. Caux, T. Barthel, U. Schollwöck, S. E. Nagler, and C. D. Frost, Multispinon Continua at Zero and Finite Temperature in a Near-Ideal Heisenberg Chain, Phys. Rev. Lett. 111, 137205 (2013).

[5] M. Mourigal, M. Enderle, A. Klöpperpieper, J. S. Caux, A. Stunault, and H. M. Rønnow, Fractional spinon excitations in the quantum Heisenberg antiferromagnetic chain, Nat. Phys. 9, 435 (2013).

[6] J.-J. Wen, S. M. Koohpayeh, K. A. Ross, B. A. Trump, T. M. McQueen, K. Kimura, S. Nakatsuji, Y. Qiu, D. M. Pajerowski, J. R. D. Copley, and C. L. Broholm, Disordered Route to the Coulomb Quantum Spin Liquid: Random Transverse Fields on Spin Ice in $\mathrm{Pr}_{2} \mathrm{Zr}_{2} \mathrm{O}_{7}$, Phys. Rev. Lett. 118, 107206 (2017).

[7] B. Gao et al., Experimental signatures of a threedimensional quantum spin liquid in effective spin-1/2 $\mathrm{Ce}_{2} \mathrm{Zr}_{2} \mathrm{O}_{7}$ pyrochlore, Nat. Phys. 15, 1052 (2019).

[8] K. W. Plumb, H. J. Changlani, A. Scheie, S. Zhang, J. W. Krizan, J. A. Rodriguez-Rivera, Y. Qiu, B. Winn, R. J. Cava, and C. L. Broholm, Continuum of quantum fluctuations in a three-dimensional $S=1$ Heisenberg magnet, Nat. Phys. 15, 54 (2019).

[9] Y. Okamoto, M. Nohara, H. Aruga-Katori, and H. Takagi, Spin-Liquid State in the $S=1 / 2$ Hyperkagome Antiferromagnet $\mathrm{Na}_{4} \mathrm{Ir}_{3} \mathrm{O}_{8}$, Phys. Rev. Lett. 99, 137207 (2007).

[10] S. Chillal, Y. Iqbal, H. O. Jeschke, J. A. Rodriguez-Rivera, R. Bewley, P. Manuel, D. Khalyavin, P. Steffens, P. Thomale, A. T. M. Nazmul Islam, J. Reuther, and B. Lake, Evidence for a three-dimensional quantum spin liquid in $\mathrm{PbCuTe}_{2} \mathrm{O}_{6}$, Nat. Commun. 11, 2348 (2020).

[11] C. Pfleiderer, G. J. McMullan, S. R. Julian, and G. G. Lonzarich, Magnetic quantum phase transition in $\mathrm{MnSi}$ under hydrostatic pressure, Phys. Rev. B 55, 8330 (1997).

[12] C. Pfleiderer, S. R. Julian, and G. G. Lonzarich, Non-Fermiliquid nature of the normal state of itinerant-electron ferromagnets, Nature (London) 414, 427 (2001).

[13] J. M. Hopkinson and H.-Y. Kee, Geometric frustration inherent to the trillium lattice, a sublattice of the B20 structure, Phys. Rev. B 74, 224441 (2006).

[14] S. V. Isakov, J. M. Hopkinson, and H. Y. Kee, Fate of partial order on trillium and distorted windmill lattices, Phys. Rev. B 78, 014404 (2008).

[15] See Supplemental Material, which includes Refs. [16-25], at http://link.aps.org/supplemental/10.1103/PhysRevLett .127.157204 for additional information on sample preparation, experimental and computational methods, powder diffraction, crystal structure data, the magnetization, specific heat, neutron scattering, and muon spin relaxation experiments, and pseudofermion functional renormalization group calculations.

[16] K. Koepernik and H. Eschrig, Full-potential nonorthogonal local-orbital minimum-basis band-structure scheme, Phys. Rev. B 59, 1743 (1999). 
[17] J. P. Perdew, K. Burke, and M. Ernzerhof, Generalized Gradient Approximation Made Simple, Phys. Rev. Lett. 77, 3865 (1996).

[18] A. I. Liechtenstein, V. I. Anisimov, and J. Zaanen, Densityfunctional theory and strong interactions: Orbital ordering in Mott-Hubbard insulators, Phys. Rev. B 52, R5467 (1995).

[19] H. O. Jeschke, F. Salvat-Pujol, E. Gati, N. H. Hoang, B. Wolf, M. Lang, J. A. Schlueter, and R. Valentí, Barlowite as a canted antiferromagnet: Theory and experiment, Phys. Rev. B 92, 094417 (2015).

[20] H. O. Jeschke, H. Nakano, and T. Sakai, From kagome strip to kagome lattice: Realizations of frustrated $S=\frac{1}{2}$ antiferromagnets in Ti(III) fluorides, Phys. Rev. B 99, 140410(R) (2019).

[21] T. Mizokawa and A. Fujimori, Electronic structure and orbital ordering in perovskite-type $3 d$ transition-metal oxides studied by Hartree-Fock band-structure calculations, Phys. Rev. B 54, 5368 (1996).

[22] J. Reuther and P. Wölfle, $J_{1}-J_{2}$ frustrated twodimensional Heisenberg model: Random phase approximation and functional renormalization group, Phys. Rev. B 81, 144410 (2010).

[23] M. L. Baez and J. Reuther, Numerical treatment of spin systems with unrestricted spin length $S$ : A functional renormalization group study, Phys. Rev. B 96, 045144 (2017).

[24] J. Polchinski, Renormalization and effective Lagrangians, Nucl. Phys. B231, 269 (1984).

[25] C. Wetterich, Exact evolution equation for the effective potential, Phys. Lett. B 301, 90 (1993).

[26] A. P. Ramirez, B. Hessen, and M. Winklemann, Entropy Balance and Evidence for Local Spin Singlets in a Kagomé-Like Magnet, Phys. Rev. Lett. 84, 2957 (2000).

[27] S. Nakatsuji, Y. Nambu, H. Tonomura, S. Sakai, O. Jonas, C. Broholm, H. Tsunetsugu, Y. Qiu, and Y. Maeno, Spin disorder on a triangular lattice, Science 309, 1697 (2005).

[28] H. J. Silverstein, K. Fritsch, F. Flicker, A. M. Hallas, J. S. Gardner, Y. Qiu, G. Ehlers, A. T. Savici, Z. Yamani, K. A. Ross, B. D. Gaulin, M. J. P. Gingras, J. A. M. Paddison, K. Foyevtsova, R. Valenti, F. Hawthorne, C. R. Wiebe, and H. D. Zhou, Liquidlike correlations in single-crystalline $\mathrm{Y}_{2} \mathrm{Mo}_{2} \mathrm{O}_{7}$ : An unconventional spin glass, Phys. Rev. B 89, 054433 (2014).
[29] J. Boerio-Goates, J. I. Artman, and B. F. Woodfield, Heat capacity studies of phase transitions in Langbeinites II. $\mathrm{K}_{2} \mathrm{Mg}_{2}\left(\mathrm{SO}_{4}\right)_{3}$, Phys. Chem. Miner. 17, 173 (1990).

[30] Y. Xu, J. Zhang, Y. S. Li, Y. J. Yu, X. C. Hong, Q. M. Zhang, and S. Y. Li, Absence of Magnetic Thermal Conductivity in the Quantum Spin-Liquid Candidate $\mathrm{YbMgGaO}_{4}$, Phys. Rev. Lett. 117, 267202 (2016).

[31] P. Mendels, F. Bert, M. A. de Vries, A. Olariu, A. Harrison, F. Duc, J. C. Trombe, J. S. Lord, A. Amato, and C. Baines, Quantum Magnetism in the Paratacamite Family: Towards an Ideal Kagome Lattice, Phys. Rev. Lett. 98, 077204 (2007).

[32] C. Balz, B. Lake, J. Reuther, H. Luetkens, R. Schönemann, T. Herrmannsdörfer, Y. Singh, A. T. M. Nazmul Islam, E. M. Wheeler, J. A. Rodriguez-Rivera, T. Guidi, G. G. Simeoni, C. Baines, and H. Ryll, Physical realization of a quantum spin liquid based on a complex frustration mechanism, Nat. Phys. 12, 942 (2016).

[33] M. Fujihala, K. Morita, R. Mole, S. Mitsuda, T. Tohyama, S. Yano, D. Yu, S. Sota, T. Kuwai, A. Koda, H. Okabe, H. Lee, S. Itoh, T. Hawai, T. masuda, H. Sagayama, A. Matsuo, K. Kindo, S. Ohira-Kawamura, and K. Nakajima, Gapless spin liquid in a square-kagome lattice antiferromagnet, Nat. Commun. 11, 3429 (2020).

[34] Y. J. Uemura, A. Keren, K. Kojima, L. P. Le, G. M. Luke, W. D. Wu, Y. Ajiro, T. Asano, Y. Kuriyama, M. Mekata, H. Kikuchi, and K. Kakurai, Spin Fluctuations in Frustrated Kagome Lattice System $\mathrm{SrCr}_{8} \mathrm{Ga}_{4} \mathrm{O}_{19}$ Studied by Muon Spin Relaxation, Phys. Rev. Lett. 73, 3306 (1994).

[35] C. Rüegg, B. Normand, M. Matsumoto, A. Furrer, D. F. McMorrow, K. W. Krämer, H. U. Güdel, S. N. Gvasaliya, H. Mutka, and M. Boehm, Quantum Magnets under Pressure: Controlling Elementary Excitations in $\mathrm{TlCuCl}_{3}$, Phys. Rev. Lett. 100, 205701 (2008).

[36] S. Mühlbauer, B. Binz, F. Jonietz, C. Pfleiderer, A. Rosch, A. Neubauer, R. Georgii, and P. Böni, Skyrmion lattice in a chiral magnet, Science 323, 915 (2009).

[37] S. Seki, X. Z. Yu, S. Ishiwata, and Y. Tokura, Observation of skyrmions in a multiferroic material, Science 336, 198 (2012).

[38] A. Kitaev, Anyons in an exactly solved model and beyond, Ann. Phys. (Amsterdam) 321, 2 (2006).

[39] https://doi.org/10.5281/zenodo.5497601. 